\title{
Allelic variation at the rpv1 locus controls partial resistance to Plum pox virus infection in Arabidopsis thaliana
}

S. Poque ${ }^{1,2,6}$, G. Pagny $^{1,2}$, L. Ouibrahim ${ }^{3}$, A. Chague ${ }^{1,2}$, J-P Eyquard ${ }^{1,2}$, M. Caballero ${ }^{1,2}$, T. Candresse ${ }^{1,2}$, C. Caranta ${ }^{3}$, S. Mariette ${ }^{1,4,5}$ and V. Decroocq ${ }^{1,2^{*}}$

\begin{abstract}
Background: Sharka is caused by Plum pox virus (PPV) in stone fruit trees. In orchards, the virus is transmitted by aphids and by grafting. In Arabidopsis, PPV is transferred by mechanical inoculation, by biolistics and by agroinoculation with infectious CDNA clones. Partial resistance to PPV has been observed in the Cvi-1 and Col-0 Arabidopsis accessions and is characterized by a tendency to escape systemic infection. Indeed, only one third of the plants are infected following inoculation, in comparison with the susceptible Ler accession.

Results: Genetic analysis showed this partial resistance to be monogenic or digenic depending on the allelic configuration and recessive. It is detected when inoculating mechanically but is overcome when using biolistic or agroinoculation. A genome-wide association analysis was performed using multiparental lines and 147 Arabidopsis accessions. It identified a major genomic region, rpv1. Fine mapping led to the positioning of rpv1 to a $200 \mathrm{~kb}$ interval on the long arm of chromosome 1. A candidate gene approach identified the chloroplast phosphoglycerate kinase ( $C P G K 2)$ as a potential gene underlying the resistance. A virus-induced gene silencing strategy was used to knock-down CPGK2 expression, resulting in drastically reduced PPV accumulation.

Conclusion: These results indicate that rpv1 resistance to PPV carried by the Cvi-1 and Col-0 accessions is linked to allelic variations at the Arabidopsis CPGK2 locus, leading to incomplete, compatible interaction with the virus.
\end{abstract}

Keywords: Partial resistance, recessive resistance, QTL mapping, association mapping, PPV, Plum pox virus, Arabidopsis thaliana, CPGK

\section{Background}

Potyviruses represent about $20 \%$ of known plant viruses and are economically among the most important threat for vegetable and fruit trees crop species. Among them, Plum pox virus (PPV) infects Prunus species (stone fruits) and causes sharka disease which devastates fruit and plant production, significantly impacting crop quality and yield. Over the last 30 years, Sharka costs to the industry worldwide have been estimated at 10 billion Euros [1]. Unfortunately, only a few sources of natural resistance are available in Prunus hosts. In

\footnotetext{
* Correspondence: decroocq@bordeaux.inra.fr

'INRA, UMR 1332 Biologie du Fruit et Pathologie, F-33140 Villenave d'Ornon, cedex, France

2Université de Bordeaux, UMR 1332 Biologie du Fruit et Pathologie, F-33140 Villenave d'Ornon, cedex, France

Full list of author information is available at the end of the article
}

order to expand the range and understand the nature of resistance sources, we are investigating new resistances to PPV in the model host plant Arabidopsis thaliana.

Arabidopsis is commonly used for the acquisition of knowledge on basic plant biology and on adaptation to biotic or abiotic stress. Its small size, rapid life cycle and small genome size of $\sim 150 \mathrm{Mb}$ make it an ideal model plant for biotechnological and genetical characterization of plant disease resistance. Moreover Arabidopsis is susceptible to various viral pathogens such as potyviruses (e.g. Turnip mosaic virus; Tobacco etch virus; Lettuce mosaic virus (LMV) or PPV), cucumoviruses (Cucumber mosaic virus), luteoviruses (Beet western yellow virus) and others, making it an ideal

\section{() Biomed Central}

(c) 2015 Poque et al. This is an Open Access article distributed under the terms of the Creative Commons Attribution License (http://creativecommons.org/licenses/by/4.0), which permits unrestricted use, distribution, and reproduction in any medium, provided the original work is properly credited. The Creative Commons Public Domain Dedication waiver (http:// creativecommons.org/publicdomain/zero/1.0/) applies to the data made available in this article, unless otherwise stated. 
host for the identification of genes underlying susceptibility or resistance to viral infection [2, 3].

Indeed, three distinct mechanisms of resistance to the PPV and LMV potyviruses have recently been identified in Arabidopsis [4-6], two of which show a recessive genetic determinism. Viruses are obligatory intracellular parasites highjacking the host cell machinery to complete the different steps of their infectious cycle. The disruption of compatible interactions between host and viral factors during replication or translation (or any other viral function) of the viral genome may lead to the failure of the corresponding infection step, operationally resulting in a recessive resistance [7]. This kind of resistance seems to be more frequent for plant/potyvirus pathosystems, representing $40 \%$ of the resistances identified, up to now, in the natural diversity of crop species. It is worth noting that most of the studies on recessive resistance to potyviruses published to date identified genes encoding the translation initiation factors eIF4E and eIF4G or their isoforms [8].

Recessive resistances against PPV and another potyvirus, Watermelon mosaic virus (WMV), were identified in the 'Cape Verde Island' Arabidopsis ecotype (Cvi-1 and Cvi-0, respectively) following mechanical inoculation. These resistances were mapped to the same interval on chromosome $1[6,9]$ and the corresponding genes were respectively named rpv1 and rwm1. In the case of rwm1, a chloroplast phosphoglycerate kinase (cPGK2) has recently been identified as responsible for WMV resistance [9]. This cytosolic isozyme of chloroplastic PGK is a ubiquitous monomeric enzyme that can also play roles in DNA repair [10] or, in the case of the paramyxovirus Sendai virus, in stimulation of mRNA transcription during the elongation step [11]. In plants, it was shown that lowering cPGK levels reduced the accumulation of Bamboo mosaic virus (BaMV), a member of Potexvirus genus [12].

Another recessive resistance against PPV has been identified among Arabidopsis accessions of diverse origins following Agrobacterium-mediated inoculation [5]. It was designated sha3 for "sharka resistance" and appears to be unlinked to rpv1 as it maps at the bottom of linkage group 3. Variation at the sha3 locus restricts PPV long distance movement and viral systemic infection [5]. In the present study, genetic analysis and linkage mapping of recombinant inbred line (RIL) populations and genome wide association mapping in a multiparental population were used to demonstrate the existence of rpv1 resistance alleles in both Cvi-1 and Col- 0 and to identify $c P G K 2$ as the cellular gene underlying this resistance to PPV in Arabidopsis. We also confirm that the rpv1-driven tendency to escape PPV infection is distinct from the sha3 resistance mechanism and that it is specific to the method of inoculation.

\section{Results}

Testing different inoculation methods on the Cvi-1, Col-0 and Ler Arabidopsis accessions

Arabidopsis thaliana can be experimentally inoculated with Plum pox virus (PPV) using different methods: i) mechanically [6, 13], ii) by biolistics [14] and iii) by agroinoculation [5]. In the first case, the virus is delivered as an encapsidated virion while purified DNA molecules are transferred by Agrobacterium or shooting. To test the effect of the inoculation method (or of the viral form) on the outcome of the Arabidopsis/PPV interactions, several accessions were inoculated in parallel with the three methods described in the Material and methods' section. The accumulation of PPV-R in the Ler, Cvi-1 and Col-0 accessions was estimated at 21 days post inoculation (dpi) by ELISA. Surprisingly, while the three accessions are fully susceptible to PPV infection after biolistic (not shown) or agroinoculation, both Cvi-1 and Col-0 showed a constant tendency to escape systemic infection when inoculated mechanically (Figure 1). Indeed, viral accumulation was detected in only 33 to $35 \%$ of inoculated plants. According to Lecocq et al. [15], partial resistance is, in some cases, based on the tendency to escape infection and may be characterized as a lower probability of infection than that of susceptible plants, using the same level of inoculum, which is the case for Cvi-1 and Col- 0 when mechanically infected by PPV. This observed phenotype in response to PPV infection will thus be named "partial resistance", hereafter.

We also checked if the phenotype observed was linked to a true, partial resistance mechanism or if the infected plants carried a PPV variant that had evolved the ability to overcome the Col-0 resistance. We carried out serial passage of PPV into Col- 0 as described in the material and methods section. In the two replicate experiments, PPV infection rate in the back inoculated Col-0 plants reached only 33.3 to $37.5 \%$, demonstrating the stability of the resistance phenotype. It thus appears that the partial block in PPV systemic infection in Arabidopsis dependent on the inoculation method is stable, and that this partial resistance in Cvi-1 and Col-0 is observed only when plants are mechanically inoculated. This resistance is overcome upon biolistic or Agrobacterium-mediated inoculation. Cvi-1 had previously been shown to be resistant to PPV upon mechanical inoculation and the locus involved, rpv1, mapped to a chromosome 1 interval [6].

\section{Genome Wide Association mapping of Arabidopsis resistance after mechanical inoculation}

We recently showed using a larger set of Arabidopsis accessions that resistance to PPV inoculated by Agrobacterium is controlled by a different locus, named sha 3 gene 


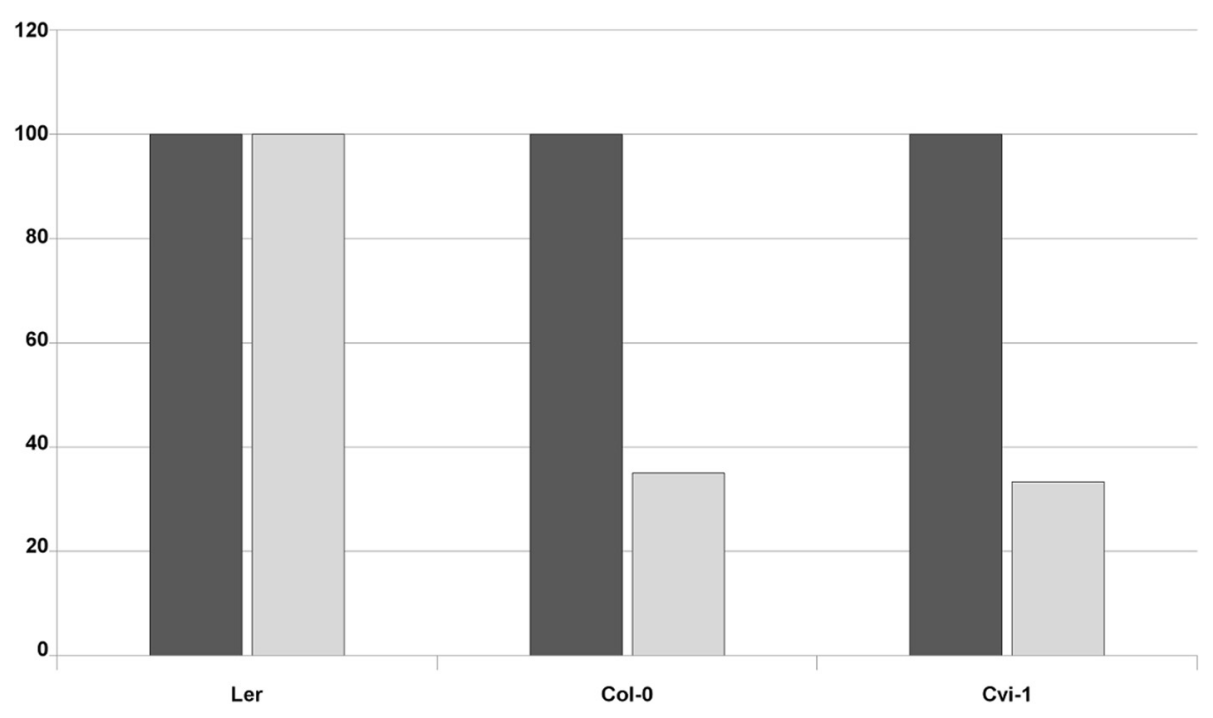

Fig. 1 Percentage of infected plants among susceptible (Ler) and partially resistant (Col-0, Cvi-1) accessions following agroinoculation (dark bars) or mechanical inoculation (light bars). The results presented are those of representative experiments involving 12 to 24 Arabidopsis plants per condition. The infection status was determined by an ELISA assays performed on non-inoculated tissues 21 days post inoculation

[5]. In an effort to evaluate that the rpv1 resistance is unrelated to the sha3 PPV resistance locus, a set of 147 Arabidopsis accessions (see Additional file 1: Table S1) previously used to identify sha3 was mechanically inoculated with PPV. The experiment was duplicated and the broad-sense heritability, calculated as described in material and methods, of PPV resistance reached 0.82 and 0.83 , respectively.

Fisher's exact test identified SNPs significantly linked to resistance to PPV. Notably, 15 of the 500 SNPs (Additional file 2: Table S2A to S2D) with the lowest $p$-values were located on chromosome one. This region coincides with a previously identified locus associated to resistance to PPV mechanical inoculation in the Cvi-1 accession and named rpv1 [6]. When using the quantitative data (normalized optical density values, see material and methods), 43, 31 and 31 SNPs, out of the 500 SNPs with the lowest $p$-values, belong to the same rpv1 genomic region, with the Wilcoxon, PLINK and EMMA methods, respectively (Additional file 2: Table $\mathrm{S} 2 \mathrm{~B}, \mathrm{C}$ and D). In addition, SNPs localized to the sha3 interval were also detected among the 500 most significant SNPs, thereby confirming that both rpv1- and sha3-driven resistance mechanisms are present, concomitantly, in the population of natural Arabidopsis accessions. However, since we cannot rule out that spurious, false positive association may arise from population structure, a traditional linkage mapping in recombinant inbred line populations was conducted, in order to confirm and fine map rpv1.
Linkage mapping of resistance to PPV systemic accumulation after mechanical inoculation of an Arabidopsis multiparental recombinant population

Four hundred and thirty-five of the 527 MAGIC (Multiparent Advanced Generation Inter-Cross) recombinant inbred lines described by Kover et al. [16] were evaluated in a three random blocks design following a mechanical inoculation. A significant block effect was detected and a QTL mapping analysis was performed using data from each block separately, as well as using a lsmeans model, accounting for the effect of each block. The broad-sense heritability of PPV resistance for the MAGIC lines was calculated from the variance analysis (see material and methods) and reached 0.77 . Interestingly, the same genomic region was identified when using data from block one and three separately or from the mean values of the three blocks. Analysis of the variation in susceptibility to PPV infection for the first and third block identified one QTL on linkage group 1 at position $19,778,790$ bp $(-\log 10 \quad(p$-value $)=4.07)$ and $22,286,231$ bp $(-\log 10$ $(p$-value $)=3.62)$, respectively. By using lsmeans values, a major QTL was identified in the same genomic region as for blocks 1 and 3, with a maxima of the $-\log 10$ ( $p$-value) of 5.80 (Table 1A and Fig. 2). This region coincides with rpv1. Surprisingly, even if point wise $p$-values were significant at the $1 \%$ level, this analysis failed to identify the sha3 PPV resistance locus which had previously been identified in the same MAGIC RILs population using Agrobacterium-mediated PPV inoculation [5]. From the QTL analysis of the MAGIC lines, the 
Table 1 Identification of Arabidopsis genomic regions controlling restriction of PPV infection in bi- and multi-parental populations

\begin{tabular}{|c|c|c|c|c|c|c|c|c|c|c|c|}
\hline \multicolumn{12}{|l|}{ A) } \\
\hline $\begin{array}{l}\text { Multiparental } \\
\text { progeny }\end{array}$ & \multicolumn{2}{|l|}{$\begin{array}{l}\text { Type of population/ } \\
\mathrm{Nb} \text { of RILs }\end{array}$} & \multicolumn{2}{|c|}{$\begin{array}{l}\text { Set of markers } \\
\text { used for the } \\
\text { analysis } \dagger\end{array}$} & $\begin{array}{l}\text { Linkage } \\
\text { group }\end{array}$ & Peak in Bp & Peak SNP & Interval in Bp & $\log P$ & \multicolumn{2}{|c|}{ Genome-wide P-Value } \\
\hline$\overline{M A G I C}$ & \multicolumn{2}{|l|}{$\mathrm{RIL} / 435^{1}$} & \multicolumn{2}{|c|}{1,260 SNPs } & LG1 & $21,665,899$ & MN1_21669564 & $\begin{array}{l}19,515,673- \\
22,286,231\end{array}$ & 5.80 & \multicolumn{2}{|l|}{0.002} \\
\hline \multicolumn{12}{|l|}{ B) } \\
\hline \multirow[t]{2}{*}{$\begin{array}{l}\text { Bi- parental } \\
\text { progenies }\end{array}$} & \multirow[t]{2}{*}{$\begin{array}{l}\text { Type of population/ } \\
\mathrm{Nb} \text { of RILs }\end{array}$} & \multirow[t]{2}{*}{$\begin{array}{l}\mathrm{Nb} \\
\text { Markers } †\end{array}$} & \multicolumn{2}{|c|}{$\begin{array}{l}\text { Parental } \\
\text { phenotypes }\end{array}$} & \multicolumn{2}{|c|}{$\begin{array}{l}\text { Predicted locus } \\
\text { location }\end{array}$} & & & & & \\
\hline & & & $\begin{array}{l}\text { Parents } \\
1\end{array}$ & $\begin{array}{l}\text { Parents } \\
2\end{array}$ & $\begin{array}{l}\text { linkage } \\
\text { group }\end{array}$ & $\begin{array}{l}\text { Resistant parental } \\
\text { allele }\end{array}$ & Marker interval & Interval in bp & $\begin{array}{l}\text { Maximum LOD } \\
(\mathrm{IM}) \neq^{*}\end{array}$ & $\begin{array}{l}\text { P-Value (Cavatorta } \\
\text { et al.) } \neq^{*}\end{array}$ & $R^{2}$ \\
\hline JEAxCol-0 & RIL F8/188' & 87 & $\mathrm{~s}$ & $\mathrm{R}$ & LG1 & Col-0 & $\begin{array}{l}\text { C1_19478/ } \\
\text { C1_23381 }\end{array}$ & $\begin{array}{l}19,477,618- \\
23,381,469\end{array}$ & 15.99 & 65.48 & $33.10 \%$ \\
\hline JEAxCol-0 & RIL F8/120 & 87 & $\mathrm{~S}$ & $\mathrm{R}$ & LG1 & Col-0 & $\begin{array}{l}\text { C1_19478/ } \\
\text { C1_23381 }\end{array}$ & $\begin{array}{l}19,477,618- \\
23,381,469\end{array}$ & 12.5 & 45.431 & $38.90 \%$ \\
\hline JEAxCol-0 & RIL F8/250' & 97 & S & $\mathrm{R}$ & LG1 & Col-0 & $\begin{array}{l}\text { F6D8-SSLP1/ } \\
\text { RCVI-32 }\end{array}$ & $\begin{array}{l}19,624,624- \\
22,181,333\end{array}$ & 21.78 & 81.865 & $34 \%$ \\
\hline
\end{tabular}

${ }_{1}$ In four repeats for the JEA $\times$ Col-0 RILs population and triplicates for the MAGIC lines. + Number of markers used to build the core genetic map (SSR or SNP) [32]. \# Detected by Interval Mapping -IM- or Krustal Wallis $-K W$-. * Significative after 1,000 permutations and at $95 \%$ statistical confidence. LogP is equivalent to the -log10( $p$-value). bp: base pairs, Maximum LOD: score associated with the peak of the LOD plot using Map QTL,

$\mathrm{R}^{2}$ : Proportion of the phenotypic variation explained by the peak of the LOD plot using multiple OTL mapping (explained variance) 


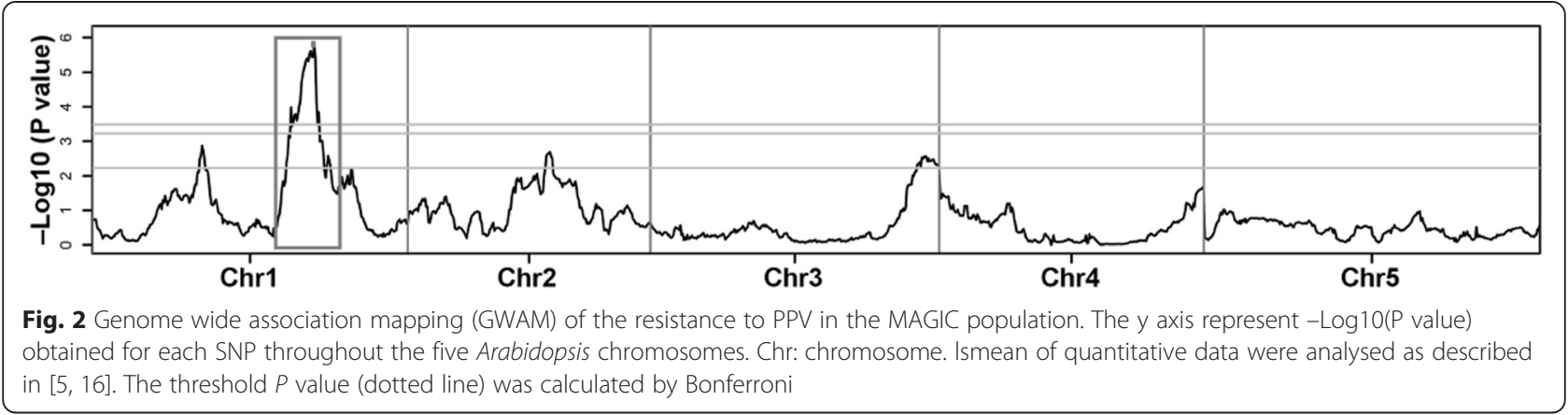

genome of each line was reconstructed as a mosaic of the founder haplotypes [16]. Based on this reconstruction, it was possible to determine that the three founders contributing the QTL detected in the MAGIC lines were Col-0, Can-0 and Ws. In comparison, PPV agroinoculation of the same MAGIC lines had shown that the sha3 resistance was contributed by two unrelated founders, Hi-0 and Sf-2 [5].

These results therefore suggest that different resistance genes may be uncovered when using different inoculation methods. The differences observed using the two inoculation methods could be due to either 1) a larger genetic effect of rpv1 over sha 3 that would hide the relative effect of the second mechanism when using mechanical inoculation, 2) a loss of the sha3-driven resistance when using mechanical inoculation or, alternatively, a loss of the rpv1-driven resistance upon agroinoculation, or 3) a difference in timing, i.e. rpv1-driven resistance taking place earlier in the viral life cycle than the sha3-driven mechanism. In order to test the first hypothesis, the analysis was repeated removing the MAGIC lines that possess a higher probability of having the genotype of the resistant founders in the rpv1 region.
The recalculated point wise $p$-value at the sha 3 locus was decreased, suggesting that removing partially the effect of the rpv1 locus improved the detection of the sha3 locus (data not shown). Therefore, even if the sha3 QTL was difficult to detect when plants were mechanically inoculated, resistance to PPV systemic infection in the MAGIC lines appears to be controlled by at least two different loci, rpv1 and sha3, respectively.

\section{Linkage mapping of the rpv1-driven resistance trait in the recombinant JEAxCol-0 population}

In order to confirm the occurrence of rpv1 in the Col-0 background, two distinct sets of biparental JEA $\times$ Col-0 recombinant inbred line population were mechanically challenged with PPV, in a completely randomized design in four independent blocks. These sets were constituted of 188 and 120 individuals, respectively. The experiment on the set of 188 RILs was repeated twice over two years.

Results show that variances are not significantly heterogeneous in all experiments (Levene's test $p$-value for both sets of 188 RILs were 0.38 and 0.33 , respectively), and results of an ANOVA test confirmed that the block design had no significant effect on viral infection ( $p$-value

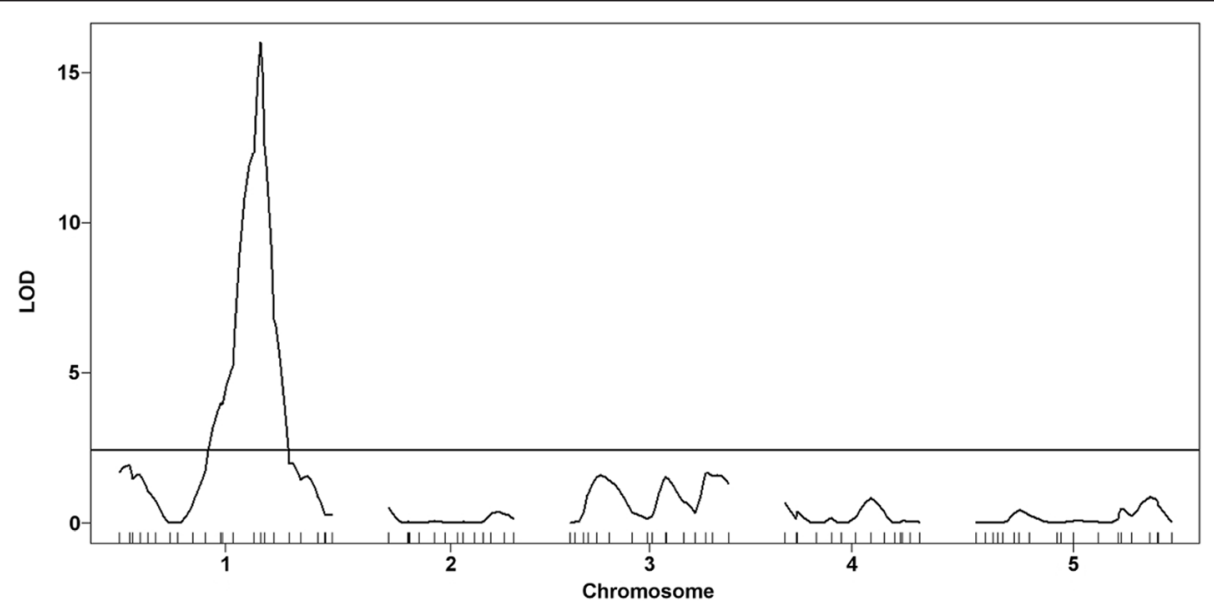

Fig. 3 Linkage mapping of the recessive resistance to PPV in an F8 JEAxCol-0 RIL population. The y axis represents the LOD score obtained by interval mapping (IM) on the first set of 188 RILS 


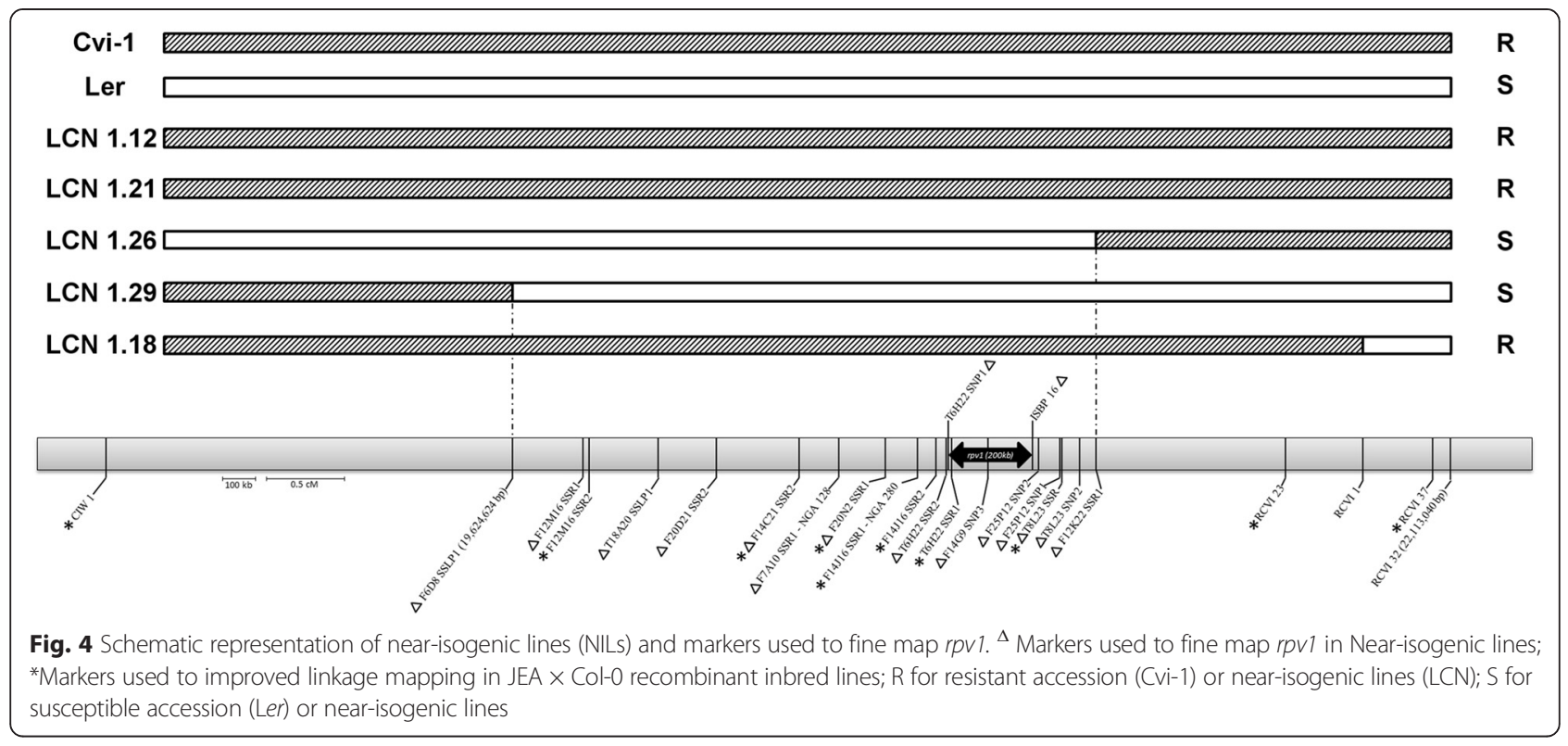

for both sets of 188 RILs were 0.3902 and 0.1898, respectively). As a consequence, the QTL analysis was performed on pooled blocks results, using the MapQTL and RQtl softwares. A Kruskal-Wallis test was first performed, to detect markers linked to the resistance to PPV. An approximate LOD score was then computed through interval mapping. Table 1B summarizes Krustal -Wallis $p$-values and LOD scores for each QTL. In the 188 RILs experiment, the effect of only one locus was observed, with a LOD score of 15.99 and a $R^{2}$ of $33.1 \%$. This single locus is located on chromosome one, between $60.5 \mathrm{cM}$ to $69.1 \mathrm{cM}$ $(19,477,618$ bp to $23,381,469$ bp) (Fig. 3), the same region previously identified in Cvi-1 [6] and in the MAGIC lines. The second set of JEA x Col-0 recombinant lines used was composed of 120 RILs selected so that they display at least one recombination event over the 60.5-69.1 cM interval identified above. Phenotyping of the set of 120 RILs resulted in the mapping of one single locus co-localizing with rpv1 (Table 1B) but with a higher effect ( $R^{2}$ up to $38.90 \%$ ).

It appears here that $\mathrm{Cvi}-1$ and $\mathrm{Col}-0$ are sharing the same genomic region. A JEA $\times$ Col-0 F1 population was tested for resistance to PPV mechanical inoculation and a majority of the plants $(75 \%)$ resulted positive. The segregation ratio in $\mathrm{F} 2 \mathrm{Cvi}-1 \times \mathrm{Ler}$ progenies [6] as well as the fact that the JEA $\times$ Col-0 F1 population is susceptible indicate that both populations display a recessive resistance to PPV. However, since the interval is still rather large, we cannot rule out, at this stage, an overlapping of two distinct loci. We thus performed the fine mapping of this region which controls resistance to PPV mechanical inoculation and allelism tests.

\section{Fine-mapping of the rpv1 locus in near isogenic backgrounds}

To avoid any epistatic interactions with other loci, the fine mapping of rpv1 was performed in near isogenic lines (NILs) originating from a Cvi-1 $\times$ Ler recombinant inbred line population [17]. The procedure was conducted in three steps as described in the material and methods section. The first step allowed us to determine the upper and lower borders of the rpv1 locus in Cvi-1 (Fig. 4), as depicted in the NILs LCN1.29 and 1.26. The identified recombination points were flanked by two markers, F6D8 SSLP1 and F12K22 SSR1 (Additional file 3: Table S3), and delineated an interval of 1.8 Mb (Fig. 4).

The second fine-mapping step allowed to reduce the rpv1 interval down to $460 \mathrm{~kb}$, between markers T6H22 SSR2 and F12K22 SSR1. The third step consisted in a second run of fine-mapping, this time using a LCN1.26 (susceptible) $\times$ LCN1.21 (resistant) F2 population of 840 individuals. In this case, all lines were screened with the T6H22 SSR2, ISBP 16, T8L23 SSR and F12K22 SSR1 markers. This allowed narrowing down the rpv1 interval to $260 \mathrm{~kb}$, between positions 20,971,975 and 21,232,895 on the long arm of chromosome 1 (Fig. 4).

The Col- 0 and Cvi-1 resistances involve the same gene In order to see if the Col- 0 and Cvi- 1 resistances involve the same gene, we performed an allelism test. Col-0 was crossed with a resistant near isogenic line carrying the 


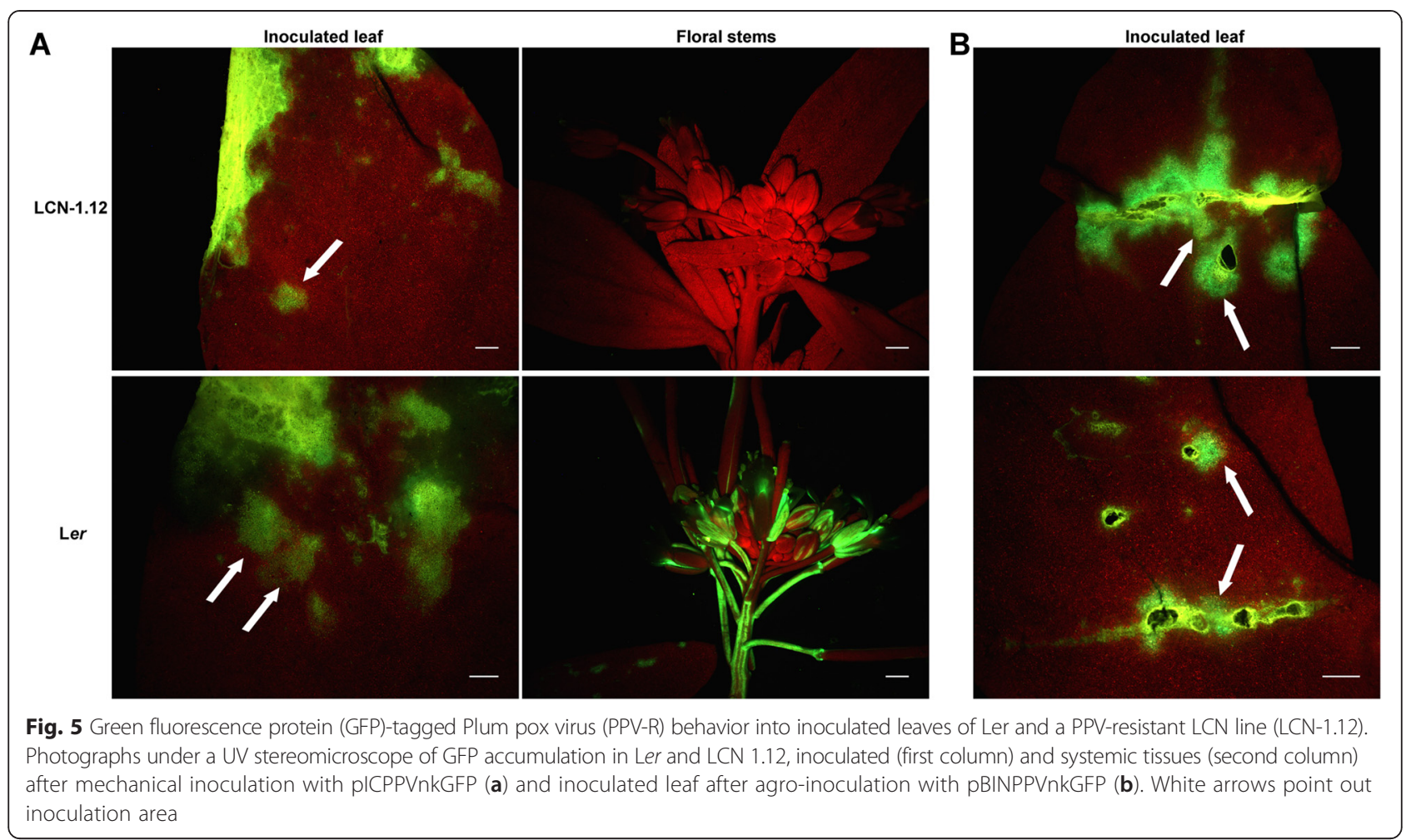

rpv1 genomic region of Cvi-1, namely LCN1.18 (See Fig. 4). The corresponding F1 progeny was mechanically challenged with PPV and $100 \%$ of tested plants were observed to be resistant. Since partial resistance in Col-0 and Cvi-1 is recessive, this allelism test demonstrates that $r p v 1$ is allelic in both accessions.

\section{Further characterization of the breakdown of the rpv1-} mediated resistance upon biolistics or agroinoculation In order to further characterize the rpv1-mediated resistance and to ensure that it had been properly mapped, Ler, Cvi-1 and ten selected LCN NILs (five PPV-resistant and five PPV-susceptible LCN near-isogenic lines) were inoculated in parallel using three techniques: mechanical inoculation, agro-inoculation or biolistics. In each case, the same viral inoculum, derived from PPV-R, was used. Mechanical inoculation of the Cvi-1, Ler and nearisogenic LCN lines provided viral infection ratios similar to those shown in Fig. 1. In comparison, when the five PPV-resistant LCN lines (LCN 1.12, 1.18, 1.21, 1.22, 1.23) were inoculated either by agroinoculation or by biolistics, $100 \%$ of the plants showed PPV systemic accumulation (not shown). Fluorescence microscopy observation of mechanically inoculated leaves of Ler and of the PPV-resistant LCN lines (see LCN1.12 as a representative example in Fig. 5A) revealed clear PPV accumulation, demonstrating that rpv1 does not prevent multiplication in inoculated leaves but only affects PPV systemic infection of non-inoculated tissues. However, an effect of $r p v 1$ on a reduction in the accumulation rate in inoculated leaves could not be ruled out.

\section{cPGK2: a potential candidate for rpv1}

Studies with the Watermelon mosaic virus (WMV) Arabidopsis thaliana pathosystem have identified in Cvi-1 a recessive resistance gene ( $r w m 1)$ that maps to the same region as the rpv1 locus. Using a combination of fine mapping and functional validation, $r w m 1$ has recently been shown to correspond to a gene coding for a chloroplast phosphoglycerate kinase (cPGK2) [9]. Given the colocalization of the $r w m 1$ and $r p v 1$ resistances, the possibility that the same $c P G K 2$ might contribute to the resistance to PPV systemic accumulation analyzed here was evaluated. Similarly to Ouibrahim et al. [9], a TRV-based VIGS system was used to knock-down $c P G K 2$ expression in $\mathrm{Ni}$ cotiana benthamiana and to evaluate its impact on PPV accumulation. The entire experiment was repeated twice and means results between these two experiments are presented in Fig. 6. The results obtained show that the levels of the chloroplast PGK2 mRNA in the PGK-5 and PGK-3inoculated plants were reduced by about $90 \%$ as compared to control plants inoculated with the PDS construct. In the same plants, PPV accumulation in systemic, non-inoculated leaves was reduced by over $90 \%$ in PGK-3- and PGK-5-silenced plants. Taken together, these results suggest that the chloroplast PGK2 is required for efficient PPV accumulation in $N$. benthamiana. 

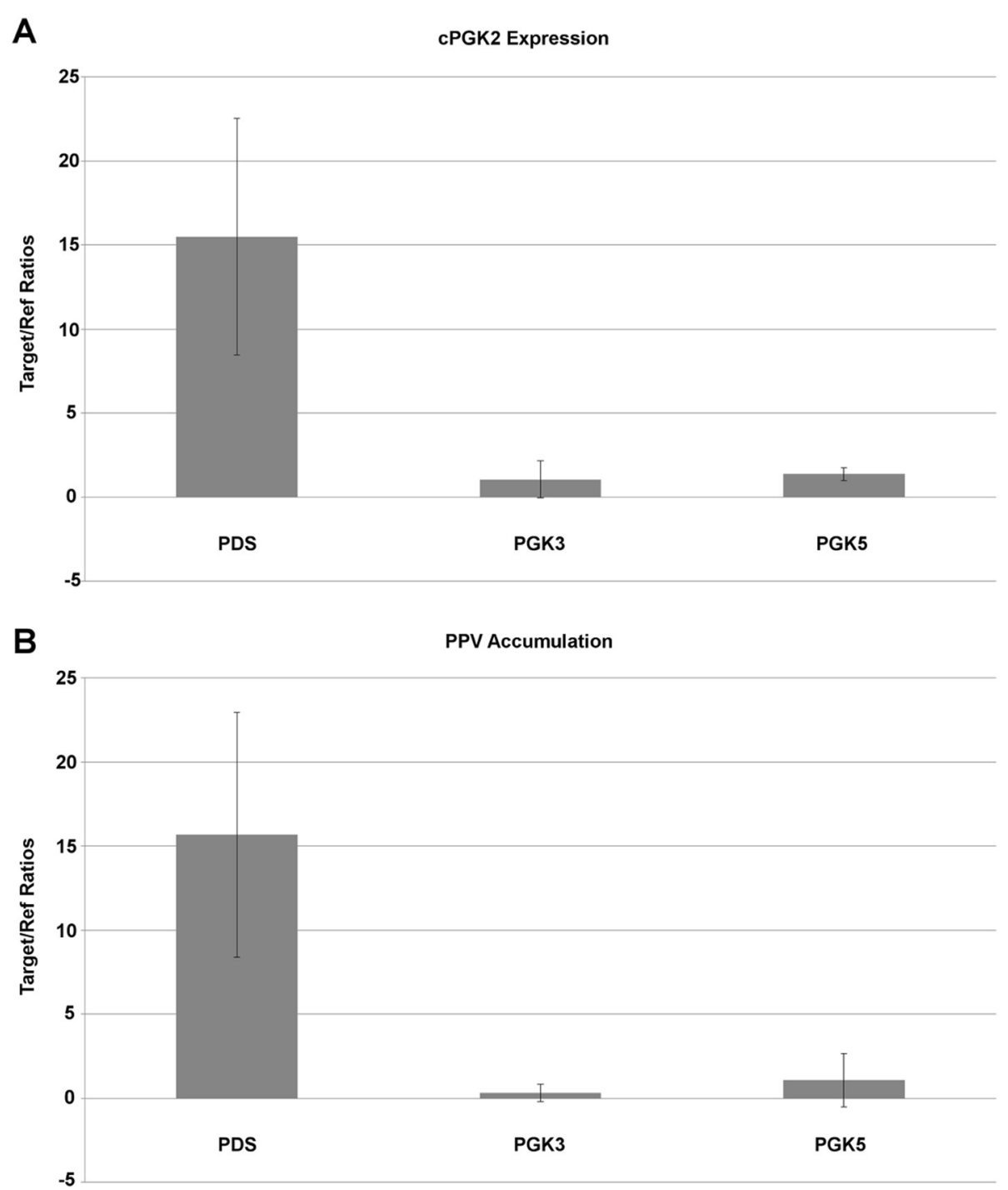

Fig. 6 Effect of the viral-induced silencing of the CPGK2 gene on PPV accumulation in Nicotiana benthamiana. a,The accumulation levels of cPGK2 transcripts and $\mathbf{b}$, PPV RNA were measured by quantitative RT-PCR in the non-inoculated leaves at 6 dpi. The values represent means ( \pm sd) of fold changes relative to the control (Mock). Each sample includes four to six biological replicates. The RNA levels were normalized to that of NbEF1. Means and standard errors are displayed as vertical bars. The phytoene desaturase (PDS) was used as positive control

\section{cPGK2 is down-expressed in Arabidopsis Col-0 rosette} leaves but not in Cvi-1.

While both Cvi-0 and Cvi-1 cPGK2 genes (At1g56190) display a non-synonymous mutation [9], no allelic difference was identified in Col-0, in comparison to Ler. We thus hypothesized that resistance to PPV in Col-0 is linked to a transcriptional regulation of the $c P G K 2$ gene. Total RNA was extracted from Col-0, Ler and Cvi-1 rosette leaves at inoculation time and $c P G K 2$ expression was tested by quantitative Real Time Reverse Transcription PCR (Q-RT-PCR) analysis. The expression level of cPGK2 was compared to the At2g36060 Arabidopsis reference gene [18] (Additional file 4: Fig. S1). Interestingly, $c P G K 2$ in Col-0 rosette leaves is two-fold downregulated in comparison with Ler and up to 14 times less expressed in comparison with Cvi-1.

\section{Discussion}

In the present study, we report the identification in Arabidopsis of a genomic region associated with partial resistance to PPV systemic accumulation upon mechanical inoculation. In order to fine map this region a combination of linkage mapping in RIL and NIL populations and of genome wide association mapping were used. Each of the bi- and multi-parental linkage mapping experiments detected a major and recurrent locus that had previously been mapped by Sicard et al. [6] and named rpv1. Allele(s) which determine this resistance 
trait are present in both the Cvi- 1 and Col- 0 accessions, as well as in Can-0 and Ws as shown in the MAGIC experiment. An allelism test indicated that restriction of PPV infection in the Cvi-1 and Col-0 accessions is controlled by the same gene. We also showed that rpv1 is distinct from the previously identified sha3 locus.

Indeed, in a recently published report, the resistance to PPV systemic accumulation following Agrobacteriummediated inoculation has been analyzed in the same set of multiparental recombinant lines, using the same viral isolate (PPV-R) [5]. This work identified loci controlling PPV systemic accumulation (in particular the sha 3 locus) but not the rpv1 locus. This could be linked to the finding that the rpv1-controlled recessive resistance is overcome when PPV is inoculated using either agroinoculation or biolistics. This phenomenon can be explained either by an over-load of PPV inoculum when using the more efficient agroinoculation or biolistics techniques, or by differences in the biological form of the virus when delivered: an encapsidated virion for mechanical inoculation, purified infectious cDNA molecules in the other techniques. Alternatively, the use of a more effective inoculation method may result in a higher number of initially infected cells or in a higher viral load in those cells, allowing the virus to overcome the rpv1 resistance mechanism. Such a scenario has been observed previously for both Cauliflower mosaic virus [19] and Plantago asiatica mosaic virus [20] but in both cases, the resistance involved was dominant. This result also poses the question of the interest and of the durability of the rpv1-driven resistance mechanism if transferred to Prunus hosts.

A recent study in WMV - Arabidopsis pathosystem identified $r w m 1$, a recessive resistance gene in Cvi-0 which co-localizes in the rpv1 interval determined here. In Cvi-0, rwm1 determines recessive resistance to WMV, with incomplete penetrance depending on the WMV isolate (up to $16 \%$ of plants are infected when infected by WMV-LL2 or -AUST89) [9]. This incomplete penetrance is speculated to be an environmental effect, as it is in particular affected by light exposure during inoculation of the plants. In the case of rpv1, the incomplete penetrance can attain a level of 33-35\% of the plants. In the experiments reported here, the different populations were tested at the same period of the year and in the same environmental conditions (e.g. same greenhouse, same time of the day for inoculations). In order to differentiate selection of resistance-breaking viral isolate from true, partial resistance, populations of PPV were allowed to evolve for 10 consecutive 21-day serial passages in the Col- 0 accession. By the end of this experiment, PPV did not show any increased virulence. Thus, rpv1 can be considered as a locus controlling partial resistance to PPV infection. The term "partial resistance" and its various interpretations are widely used in plant/pathogen genetics, in particular in describing plant/virus interactions. In most cases it has been associated with quantitative resistance and described as a reduction of disease intensity, or of pathogen accumulation, rather than the absence of disease [21, 22]. It can be attributed to a lower viral multiplication or accumulation, with the virus able to infect the host plant systemically but remaining at a lower concentration in plant tissues [23, 24]. Some authors also used the term partial resistance when viruses are restricted to specific tissues or to specific stages of the host plant development [25-27]. Finally, others describe partial resistance as an absence of symptoms despite a normal viral accumulation in systemic tissues (tolerance).

In the case of the rpv1 resistance, partial resistance to PPV infection in Cvi- 1 and Col- 0 is characterized by the tendency to escape systemic infection upon mechanical inoculation. Given the recessive nature of the resistance, this could be explained by a weaker interaction between host factor(s) and viral proteins. Among potential candidate genes present in the restricted $r w m 1$ interval, Ouibrahim et al. [9] discovered a non-synonymous mutation (S78G) in the Cvi-0 and Cvi-1 $c P G K 2$. They demonstrated, using a TRV-based virus induced gene silencing system in $N$. benthamiana, that WMV accumulation was affected by the reduction of the AT1G56190 cPGK2 transcripts. The same approach was used here to demonstrate that $c P G K 2$ expression is also necessary for efficient PPV accumulation in $N$. benthamiana systemic leaves. Therefore, a likely hypothesis is that RPV1 might be a functional $c P G K 2$ gene, in PPV susceptible accessions such as Ler. Partial resistance in the Cvi-1 accession can be potentially explained by a weaker interaction between Cvi-cPGK2 and PPV protein(s) as a result of the identified S78G mutation. However, the Col-0 $c P G K 2$ gene does not display nucleotide variation in comparison with Ler and rpv1 resistance in Col-0 might occur at another level, possibly $c P G K 2$ reduced expression. Since rpv1 is controlling a recessive resistance mechanism, susceptibility to the virus is thus dependent on the amount of cPGK2 proteins available for full compatible interaction.

In this respect, it is worth noting that in the complete Arabidopsis transcriptome data base (CATdb) the Ler cPGK2 transcript level was not significantly different between PPV-infected and mock inoculated plants (http:// urgv.evry.inra.fr/cgi-bin/projects/CATdb/consult_project.pl? project_id=118) while Babu et al. [28] showed an induction of $c$ PGK2 expression in Col-0 leaf tissues 17 days post PPV inoculation. In our case, we showed a significantly lower expression of $c P G K 2$ in Col-0 in comparison with the susceptible Ler accession. In consequence, a limitation of cPGK2 transcripts in the inoculated leaves could explain the partial resistance of Col-0 to PPV infection. However, 
while our results are in agreement with experiments conducted with WMV [9], we showed here that $c P G K 2$ gene silencing affects viral accumulation in $N$. benthamiana, not the number of plants truly infected.

\section{Conclusion}

Most of the studies on recessive resistance published to date describe alleles of genes encoding the translation initiation factors [8] as mediators of virus resistance. The present report describes a PPV recessive resistance mechanism potentially involving a chloroplast phosphoglycerate kinase. The identification of a new PPV resistance mechanism, distinct of the translation initiation complex, is important for developing novel strategies for resistance gene pyramiding in stone fruit crop species. However, deployment of such resistance specific to the inoculation method has to be considered carefully and should be combined with other, more general, resistance mechanisms.

\section{Methods}

\section{Plant material}

We used two Arabidopsis populations of recombinant inbred lines (RILs), one derived from a cross between Col-0 (Columbia) (186AV in the VNAT collection, N1092 in the NASC collection) and JEA (25AV), and the other from a cross between Cape Verde Islands (Cvi-1) and Col-0. Both of them were developed by VNAT INRA of Versailles (http://publiclines.versailles.inra.fr/).

For fine-mapping, we also used the so-called LCN near isogenics lines [17]. They originated from a Landsberg erecta (Ler) $\times$ Cvi-1 cross in which Cvi-1 genomic regions were introgressed into a Ler background. From the LCN NILs, we generated two F2 populations as follows: one by backcrossing the PPV resistant LCN 1.12 in Ler and the second by crossing the LCN1-26 (susceptible) with LCN 1.21 (resistant). F1 heterozygous plants were checked before selfing with the AthGENEA marker for the first LCN1.12 $\times$ Ler cross and with T18A20 SSLP1 for LCN1.26 $\times$ LCN1.21 (see markers listed in Additional file 3: Table S3).

Arabidopsis natural accessions for genome-wide association study and the MAGIC (Multiparent Advanced Generation Inter-Cross) recombinant population were obtained from the Nottingham Arabidopsis Stock Centre (NASC) (http://szlapncs01.nottingham.ac.uk/).

Plants were grown in a BL-3 containment greenhouse under temperature and humidity controlled conditions $\left(20{ }^{\circ} \mathrm{C}\right.$ and relative humidity of $\left.60 \%\right)$.

\section{Viral material}

Arabidopsis thaliana is inoculated with PPV using different methods: i) mechanically using an inoculum derived from pICPPVnkGFP-infected Nicotiana benthamiana leaves
$[6,13]$, ii) by biolistics using a pICPPVnkGFP cDNA clone [14] and iii) by agroinoculation with a pBINPPVnkGFP clone introduced in Agrobacterium tumefaciens [5]. In each case, the two viral clones, i.e. pICPPVnkGFP and pBINPPVnkGFP, are derived from the same PPV-R isolate, which belongs to the PPVDideron strain [29]. Construction of pBINPPVnkGFP containing the full-length nucleotide sequence of PPV$\mathrm{R}$ coupled with the green fluorescence (GFP) protein has been described by Fernández-Fernández et al. [30].The serial passage assay was done by using homogenates from PPV-positive Col-0 plants at $21 \mathrm{dpi}$ to inoculate 24 to $48 \mathrm{Col}-0$ in 10 successive passaging assays. The experiment was repeated twice, in parallel.

\section{PPV resistance phenotyping}

The pICPPVnkGFP virus clone was mechanically inoculated on the rosette leaves at four weeks after sowing. Virus infection was scored at 21 days post inoculation (dpi) in non-inoculated tissues (flower stems or newly developed rosette leaves). The inoculum was derived from pICPPVnkGFP-infected Nicotiana benthamiana leaves. At $21 \mathrm{dpi}$, viral accumulation was estimated for each individual plant using double antibody sandwich (DAS) ELISA assays [6]. Optical densities (OD) were normalized using the PPVnkGFP infected Nicotiana benthamiana positive control deposited on every ELISA plate of an assay. Quantitative data were normalized relative to the value of the PPVnkGFP infected $N$. benthamiana, which was set at 100 . In the case of RILs, the final viral accumulation value is the average of normalized measurements from all PPV-inoculated replicates of each RIL.

\section{Mapping of the genetic determinants in bi-parental populations}

The F8 JEAxCol-0 RIL population (28RV, http://publicli nes.versailles.inra.fr/rils/index) is comprised of 455 lines genotyped with 87 markers [31]. Two sets of 188 and 120 individuals, both parents, and the PPV resistant E6 eIFiso4E loss-of-function mutant [13], that served as a negative control, were challenged with PPV. Experiments were set up in a 4-blocks random design and the 188 set was duplicated over two years during the same winter period, while the next 120 RILs, recombinant over the candidate rpv1 region, were tested only once. For both data sets (188 and 121 RILs), descriptive analysis was performed under R (http://www.R-project.org).

A genetic map was constructed for the F8 JEAxCol-0 RIL population using Joinmap [32] with a LOD (logarithm of odds) score threshold of 3. Molecular markers were provided by the VNAT INRA website. Quantitative trait analysis was performed with MapQTL6 (http:// www.kyazma.nl/index.php/mc.MapQTL/) using first the 
non-parametric Kruskal-Wallis test because in both cases the distribution of the trait was not normal. Interval mapping was also performed to determine the LOD score of the putative QTLs. The percentage of the phenotypic variation explained by the QTL corresponds to the regression value $R^{2}$ taken at the peak LOD score of the QTL in the MapQTL3.

In order to fine map the locus associated with partial resistance to mechanically inoculated PPV in Cvi-1, a total of twenty molecular markers were developed (see Additional file 3: Table S3). Microsatellite repeat motifs were identified in Arabidopsis BAC sequences using Sputnik software (http://www.appliedbioinformatics.com.au/ projects/ssrPrimer/cgi-bin/index). Simple sequence length polymorphism (SSLP) and Single Nucleotide Polymorphism (SNP) were retrieved from the Monsanto Arabidopsis Polymorphism and Ler sequence collections (http://www. arabidopsis.org/browse/Cereon/index.jsp) using the name of the BAC clones as a search tag. Insertion Site-Based Polymorphism (ISBP) was identified by submitting the Col-0 genomic sequence to the ISBP Finder software [33]. Consequently, oligonucleotide primers complementary to the regions flanking the identified repeat motifs or polymorphic sites were designed using the program Primer version 0.5 (National Biosciences, Plymouth, Minnesota), setting an annealing temperature of $57.5^{\circ} \mathrm{C}$.

PCR fragments were amplified as described by Decroocq et al. [34]. SSR and SSLP markers were separated on $4 \%$ agarose gels while SNP and ISBP markers were scored by High Resolution Melting curve (HRM) on a real-time PCR LightCycler. Three of the above markers (namely T18A20 SSLP1, F7A10 SSR1 and T6H22 SSR2) were tested in the F2 LCN1.12 $\times$ Ler population. The forward primer was labeled either with FAM or with VIC and the allelic pattern of the three markers was scored among 1,736 F2 individuals in triplex on a capillary $3730 \mathrm{ABI}$ sequencing machine.

To align the Cvi-1 $\times$ Ler and JEA $\times$ Col-0 genetic maps, we selected from the above markers seven SSR and SSLP markers co-localizing with the $r p v 1$ locus and polymorphic between the JEA and Col-0 parental accessions (see Fig. 1). Two markers flanking the JEA $\times$ Col-0 locus were added, namely CIW1 and RCVI37. CIW1 is derived from the MSAT database (http:// www.inra.fr/internet/Produits/vast/msat.php). RCVI37 is a microsatellite marker designed from the T7P1 BAC sequence, as described above (Additional file 3: Table S3). All 9 markers were screened on the full set of 250 JEA $\times$ Col-0 RILs before rerunning the quantitative analysis as described above.

\section{Fine-mapping of the $r p v 1$ locus}

The fine mapping was conducted in three steps. First, a set of ten NILs, recombinant over the long arm of linkage group one, was challenged by mechanical inoculation. Then we pursued by screening 1,732 F2 plants from the LCN1.12 $\times$ Ler cross with a set of polymorphic markers developed specifically over the rpv1 interval determined above (Additional file 3: Table S3). Identified F2 recombinant individuals were self-pollinated and twelve to twenty-four F3 plants per F2 recombinant were challenged with PPV by mechanical inoculation. Because $r p v 1$ determines partial resistance to PPV infection, F3 lines having over $70 \%$ of plants in which no PPV accumulation was observed were scored as resistant. The third step consisted in a second run of fine-mapping, this time using a LCN1.26 (susceptible) $\times$ LCN1.21 (resistant) F2 population of 840 individuals.

\section{Mapping of the genetic determinants in the multiparental population (MAGIC lines)}

Four hundred and thirty five of the original set of 527 Multiparent Advanced Generation Inter-Cross (MAGIC) recombinant lines were tested in triplicates, following a complete random 3-block design. Included in these blocks were the 19 founders of the MAGIC lines and the PPV resistant E6 mutant. PPV accumulation in each recombinant line and founder was quantified by serological tests. Optical density values (OD) were scored as described above they were later used for quantitative analysis. The heritability among MAGIC lines was determined to best fit a random effects model $[17,16]$. Using ANOVA, we determined the specific effect of 'genotype' and the broad-sense heritability $\left(h^{2}\right)$, which is the ratio between the genetic variance and the total phenotypic variance was calculated using the formula $h^{2}=\sigma_{g}^{2} /\left[\sigma_{g}^{2}+\left(\sigma_{e}^{2} / n\right)\right]$, where $\sigma_{g}^{2}$ is the genetic variance, $\sigma_{e}^{2}$ is the environmental variance and $n$ the number of replicates. We estimated the phenotypic mean by using an lsmean model expressed as $l s_{\mathrm{i}}=\mu_{\mathrm{i}}+L+B$ where $l s$ was the lsmean value of each lines (i), $\mu$ represents the mean of viral accumulation for each lines (i), $L$ and $B$ were estimations of the lines and block effects, respectively. Procedures for statistic and QTL analyses are described elsewhere $[16,5]$.

\section{Association mapping}

One hundred and forty-seven accessions previously genotyped with 216,000 SNPs [35] were mechanically inoculated following a complete random 4-block design, the experiment was repeated over two years. The broadsense heritability $\left(h^{2}\right)$ was evaluated as described above. Quantitative data for viral accumulation and the estimation of the phenotypic mean (lsmean model) were generated as described for the MAGIC lines. We chose to combine the two experiments by considering the 8 blocks as a single data set in the lsmean model. To resolve the phenotypic scoring into binary data, thus 
avoiding intermediate scores, we decided to rate accessions as susceptible (an assigned value of 1 ) when the lsmean value was at least three times the lsmean value of the PPV resistant E6 negative control [13].

Genotypic data (mostly Single Nucleotide Polymorphism) were assigned as described in Atwell et al. [35] and are publically available at the AtPolyDB database (https:// easygwas.tuebingen.mpg.de/data/public/dataset/view/1/).

Fisher's exact tests were implemented on binary data to test for the association between genotypes and phenotypes. For quantitative data we used a Wilcoxon rank sum tests in addition a regression analysis was implemented using the Plink software [36] (http://pngu.mgh.harvard.edu/purcell/plink/). All those analyses were expected to have false positives due to population structure. Thus, we also used the EMMA method [37] based on a mixed model that accounts for effects due to population structure.

\section{cPGK2 Virus-Induced Gene Silencing (VIGS) in Benthamiana}

The Tobacco rattle virus (TRV) based VIGS system was used to knock down the expression of host genes. Construction of the pTRV1, pTRV2/PGK-5, pTRV2/PGK-3 and pTRV2/PDS plasmids is described in Ouibrahim et al. [9]. Two plasmids pTRV2/PGK-5 and pTRV2/ PGK-3 containing fragments corresponding to $5^{\prime}$ and $3^{\prime}$ ends of tobacco chloroplast PGK, were used to knock down the Arabidopsis AT1G56190 chloroplast PGK. Plasmid pTRV2/PDS containing a phytoene desaturase was used as a positive control of silencing [12].

Nicotiana benthamiana was grown in BL-3 containment greenhouse under the controlled conditions of temperature $\left(20{ }^{\circ} \mathrm{C}\right)$ and relative humidity $(60 \%)$. Agrabacterium tumefaciens cultures at $\mathrm{OD}_{600}=1$ containing pTRV1 or pTRV2 derivative plasmids were mixed in 1:1 ratio and infiltrated with a syringe onto two leaves of each plant. Twelve days after infiltration, newly formed leaves were mechanically inoculated with the pICPPVnkGFP clone. Six days post inoculation (dpi), newly-formed, non-inoculated leaves were sampled.

Total RNA was extracted from N. Benthamiana using the SV Total RNA Isolation System ${ }^{\circ}$ from Promega Biosciences, LLC. First strand cDNA was synthesized from total RNA using Superscript $\mathrm{II}^{\circ}$ reverse transcriptase from Invitrogen. Quantitative RT-PCR (Q-RT-PCR) was performed on a Light Cycler 480 II machine (Roche Diagnostics) by using LightCycler ${ }^{\circ}$ 480 SYBR Green I master and one tenth of the newly synthesized cDNAs. The chloroplast-specific PGK was amplified and detected by a forward primer ( $5^{\prime}$ GCCTTCTGTTGCAGGTTTCC-3') and a reverse primer (5' ${ }^{\prime}$ ATTCCTCCACCCAAAAGCAA-3'). PCR was performed using the following cycling conditions: $95{ }^{\circ} \mathrm{C}$ for $5 \mathrm{~min}$, and 40 cycles of $95{ }^{\circ} \mathrm{C}$ for $30 \mathrm{~s}, 60{ }^{\circ} \mathrm{C}$ for $30 \mathrm{~s}$ and $72{ }^{\circ} \mathrm{C}$ for $45 \mathrm{~s}$ followed by a melting curve ramp from $72{ }^{\circ} \mathrm{C}$ to $95{ }^{\circ} \mathrm{C}$ for $10 \mathrm{~s}$. Q-RT-PCR experiment was conducted on four to six biological replicates for each sample. For comparison of the data among experimental samples, the real-time PCR results were normalized using the levels of $N$. benthamiana elongation factor 1 (NbEF1) mRNA using a forward primer (5'GATTGGTGGTATTGGAACTGTC-3') and a reverse primer (5'-AGCTTCGTGGTGCATCTC-3') [38].

\section{CPGK2 expression analysis in Arabidopsis}

Rosette leaves of Ler, Col-0 and Cvi-1 were harvested just before inoculation. RNA was isolated by using the Macherey Nucleospin Total RNA isolation kit. After RNA extraction, RNA was diluted to 50 to $100 \mathrm{ng} / \mu \mathrm{L}$ with DEPC treated water. Reverse transcription was done by using $0.5 \mu \mathrm{g}$ of total RNA and Revertaid/Ribolock reverse transcriptase kit (Fermentas). Q-RT-PCR was performed as described above. The Arabidopsis cPGK2 primers used for Q-RT-PCR analysis were: forward CCTCCTTTGGACACATTCCC and reverse ATCTCCAACACTCTTCTTCGC. PCR was performed using the following cycling conditions: $95{ }^{\circ} \mathrm{C}$ for $5 \mathrm{~min}$, and 40 cycles of $95{ }^{\circ} \mathrm{C}$ for $30 \mathrm{~s}, 59^{\circ} \mathrm{C}$ for $30 \mathrm{~s}$ and $72{ }^{\circ} \mathrm{C}$ for $45 \mathrm{~s}$ followed by a melting curve ramp from $72{ }^{\circ} \mathrm{C}$ to $95{ }^{\circ} \mathrm{C}$ for $10 \mathrm{~s}$. Two independent Q-RT-PCR experiments were conducted, testing three plants per accession in each experiment.

Relative expression was calculated using the Efficiency method (Roche Diagnostics) in comparison with the At2g36060 endogenous control [19]. Fold change in $c P G K 2$ expression was determined relative to the reference gene.

\section{Additional files}

Additional file 1: Table S1. Arabidopsis accessions genotyped with the full 250 K SNPs data set and challenged with PPV by mechanical inoculation. The experiment was repeated twice and in each experiment, all accessions were tested in four replicates, following a complete random 4-block procedure.

Additional file 2: Table S2. Most informative SNPs in the 500 top markers when testing genome wide association with restricted PPV systemic infection in Arabidopsis accessions. (A) Results of the mean binary data, after Fisher analysis. (B) Results of the quantitative data after Wilcoxon rank sum test. (C) Results of the quantitative data after PLINK method. (D) Results of the quantitative data after EMMA method. *: SNPs belonging to the rpv1 interval (see Table 1). $\varphi$ : SNPs belonging to the sha3 interval [55].

Additional file 3: Table S3. Primers use to fine map rpv1 in LCN near isogenic lines and JEA x Col-0 RIL population *: Base pair.

Additional file 4: Figure S1. Relative expression of the CPGK2 (At1g56190) gene in Arabidopsis accessions. The relative expression of the Arabidopsis CPGK2 gene (At1g56190) in rosette leaves, before PPV infection, was calculated in comparison with the $\mathrm{C} 34260$ reference gene corresponding to At2g36060. The experiment was repeated twice, depicted in light and dark grey colors. 


\section{Abbreviations}

Col-0: Columbia-0; CPGK: chloroplastic phosphoglycerate kinase; Cvi-0 and -1 : Cape Verde Island-0 and -1; GWAS: Genome Wide Association Study; ISBP: Insertion Site-Based Polymorphism; Ler: Landsberg erecta; LOD: logarithm of odds; MAGIC: Multiparent Advanced Generation Inter-Cross; NIL: Near Isogenic Line; PPV: Plum Pox Virus; Q-RT-PCR: Quantitative Reverse Transcribed Polymerase Chain Reaction; QTL: Quantitative trait locus; RIL: Recombinant Inbred Line; SNP: Single Nucleotide Polymorphism; SSLP: Simple sequence length polymorphism; VIGS: Virus-induced gene silencing.

\section{Competing interest}

INRA (represented by L.O, C.C and V.D.) together with GENOPLANT VALOR hold a patent related to the use of the chloroplastic phosphoglycerate kinase (CPGK2) in anti-viral strategies (reference MJP/ahF1516/40WO1).

\section{Authors' contributions}

V.D. and S.P. conceived and designed the experiments with the contribution of T.C.; G.P., V.D. and S.P. performed the experiments; G.P., P.P. and V.D. developed molecular markers and mapping tools; J.P.E. and A.C. contributed reagents/material/ELISA analysis and M.C. to Q-RT-PCR analysis; V.D., and S.P. performed the QTL analysis; S.M. analyzed the data for association mapping and from multiparental lines; L.O. and C.C. developed VIGS constructions and provided data on candidate genes. V.D. and S.P. wrote the paper with proofreading from S.M. and T.C. All authors read and approved the final version of the manuscript.

\section{Acknowledgements}

This work was supported by the SharCo FP7 Small Collaborative Project No 204429, by grants from the EPR Aquitaine ( $\# 20091201003$ ) and INRA division of Plant Breeding. We are grateful to T. Mauduit and A. Bailly for plant production. Special thanks are given to S. Decroocq for advice in the statistical analysis. Grateful thanks to Pr. A.G. Abbott (Clemson Univ) for critical reading of the manuscript and to J-A García (CNB-CSIC, Madrid) for providing the various PPV-R infectious clones. Imaging was performed at the Bordeaux Imaging Center, member of the national infrastructure France Biolmaging.

\section{Author details}

'INRA, UMR 1332 Biologie du Fruit et Pathologie, F-33140 Villenave d'Ornon, cedex, France. ${ }^{2}$ Université de Bordeaux, UMR 1332 Biologie du Fruit et Pathologie, F-33140 Villenave d'Ornon, cedex, France. ${ }^{3}$ INRA-UR1052, Genetics and Breeding of Fruits and Vegetables, Dom. St Maurice, CS 60094, F-84143 Montfavet cedex, France. ${ }^{4}$ Current address: INRA, UMR 1202 Biogeco, F- 33610 Cestas, France. ${ }^{5}$ Current address: Univ. Bordeaux, UMR1202 Biogeco, F-33400 Talence, France. ${ }^{6}$ Current address: Department of Plant Pathology, National Chung Hsing University, Taichung 402, Taiwan.

\section{Received: 21 February 2015 Accepted: 17 June 2015}

\section{Published online: 25 June 2015}

\section{References}

1. Cambra M, Capote N, Myrta A, Llácer G. Plum pox virus and the estimated costs associated with sharka disease. EPPO Bull. 2006;36:202-4.

2. Carr T, Whitham S. An Emerging Model System: Arabidopsis as a Viral Host Plant. In: WEaH M, editor. Plant cell monographs: Viral Transport in Plants. vol. 7th ed. Berlin Heildelberg: Springer-Verlag; 2007. p. 159-83.

3. Ouibrahim L, Caranta C. Exploitation of natural genetic diversity to study plant-virus interactions: what can we learn from Arabidopsis thaliana? Mol Plant Pathol. 2013;14(8):844-54.

4. Cosson P, Sofer L, Le QH, Léger V, Schurdi-Levraud V, Whitham SA, et al. RTM3, Which Controls Long-Distance Movement of Potyviruses, Is a Member of a New Plant Gene Family Encoding a Meprin and TRAF Homology Domain-Containing Protein. Plant Physiol. 2010;154:222-32.

5. Pagny G, Paulstephenraj PS, Poque S, Sicard O, Cosson P, Eyquard J-P, et al. Family-based linkage and association mapping reveals novel genes affecting Plum pox virus infection in Arabidopsis thaliana. New Phytologist. 2012;196(3):873-86.

6. Sicard O, Loudet O, Keurentjes J-J-B, Candresse T, Le Gall O, Revers F, et al. Identification of QTLs controlling symptom development during viral infection in Arabidopsis thaliana. Mol Plant-Microbe Interact. 2008;21(2):198-207.

7. Fraser RSS. The genetics of resistance to plant viruses. Annu Rev Phytopathol. 1990;28:179-200.

8. Truniger $V$, Aranda MA. Recessive Resistance to Plant Viruses. Adv Virus Res. 2009:75:119-59.

9. Ouibrahim L, Mazier M, Estevan J, Pagny G, Decroocq V, Lecoq H, et al. Cloning of the Arabidopsis rwm1 gene for resistance to Watermelon mosaic virus points to a new function for natural virus resistance genes. Plant J. 2014;79(5):705-16.

10. Popanda O, Fox G, Thielmann H. Modulation of DNA polymerases $a, \delta$ and $\varepsilon$ by lactate dehydrogenase and 3-phosphoglycerate kinase. Biochimica et Biophysica Acta. 1998;1397:102-17.

11. Ogino T, Yamadera T, Nonaka T, Imajoh-Ohmi S, Mizumoto K. Enolase, a cellular glycolytic enzyme, is required for efficient transcription of Senda virus genome. Bio Biophys Res Commun. 2001;285(2):447-55.

12. Lin J, Ding M, Hsu Y, Tsai C. Chloroplast phosphoglycerate kinase, a gluconeogenetic enzyme, is required for efficient accumulation of Bamboo mosaic virus. Nucleic Acid Res. 2007;35:424-32

13. Decroocq V, Sicard O, Alamillo J-M, Lansac M, Eyquard J-P, García J-A, et al. Multiple resistance traits control PPV infection in Arabidopsis thaliana. Mol Plant-Microbe Interact. 2006;19:541-9.

14. Decroocq V, Salvador B, Sicard O, Glasa M, Svanella L, Cosson P, et al. The determinant of potyviruses ability to overcome the RTM resistance of Arabidopsis thaliana maps to the N-terminal region of the coat protein. Mol Plant Microbe Interact. 2009;22:1302-11.

15. Lecoq H, Moury B, Desbiez C, Palloix A, Pitrat M. Durable virus resistance in plants through conventional approaches: a challenge. Virus Res. 2004;100:31-9.

16. Kover PX, Valdar W, Trakalo J, Scarcelli N, Ehrenreich IM, Purugganan MD, et al. A Multiparent Advanced Generation Inter-Cross to Fine-Map Quantitative Traits in Arabidopsis thaliana. PLoS Genet. 2009;5(7), e1000551.

17. Keurentjes J, Bentsink L, Alonso-Blanco C, Hanhart C, Blankestijn-De Vries H, Effgen $S$, et al. Development of a Near-isogenic line population of Arabidopsis thaliana and comparison of mapping power with a recombinant inbred line population. Genetics. 2007;175:891-905.

18. Cosson P, Schurdi-Levraud V, Le Q, Sicard O, Caballero M, Roux F, et al. The RTM Resistance to Potyviruses in Arabidopsis thaliana: Natural Variation of the RTM Genes and Evidence for the Implication of Additional Genes. Plos One. 2012;7(6), e39169.

19. Callaway A, Liu W, Andrianov V, Stenzler L, Zhao J, Wettlaufer S, et al. Characterization of cauliflower mosaic virus (CaMV) resistance in virus-resistant ecotypes of Arabidopsis. Mol Plant Microbe Interact. 1996;9:9.

20. Yamaji Y, Maejima K, Komatsu K, Shiraishi T, Okano Y, Himeno M, et al. Lectin-Mediated Resistance Impairs Plant Virus Infection at the Cellular Level. Plant Cell. 2012;24:778-93.

21. Marcel T, Gorguet B, Ta M, Kohutova Z, Vels A, Niks R. Isolate specificity of quantitative trait loci for partial resistance of barley to Puccinia hordei confirmed in mapping populations and near-isogenic lines. New Phytol. 2008;177:743-55.

22. Lu Q, Bjørnstad $\AA$, Ren Y, Asad M, Xia X, Chen X, et al. Partial resistance to powdery mildew in German spring wheat "Naxos" is based on multiple genes with stable effects in diverse environments. Theor Appl Genet. 2012;125:297-309.

23. Caranta C, Palloix A, Lefebvre $V$, Daubèze AM. QTLs for a component of partial resistance to cucumber mosaic virus in pepper : restriction of virus installation in host cells. Theor Appl Genet. 1997;94:431-8.

24. García-Cano E, Navas-Castillo J, Moriones E, Fernández-Muñoz R. Resistance to Tomato chlorosis virus in Wild Tomato Species that Impair Virus Accumulation and Disease Symptom Expression. Phytopathology. 2010;100:582-92.

25. Broers L. Influence of development stage and host genotype on three components of partial resistance to leaf rust in spring wheat. Euphytica. 1989;44:187-95.

26. Headrick J, Pataky J. Expression of Partial Resistance to Common Rust in Sweet Corn Hybrids at Various Host Growth Stages. Phytopathology. 1987;77:454-8

27. Qi X, Niks R, Stam P, Lindhout P. Identification of QTLs for partial resistance to leaf rust (Puccinia hordei) in barley. Theor Appl Genet. 1998;96:1205-15.

28. Babu M, Griffiths J, Huang T-S, Wang A. Altered gene expression changes in Arabidopsis leaf tissues and protoplasts in response to Plum pox virus infection. BMC Genomics. 2008;9:325. 
29. Riechmann JL, Lain S, Garcia JA. Infectious in vitro transcripts from a plum pox potyvirus cDNA clone. Virology. 1990;177:710-6.

30. Fernández-Fernández MR, Mouriño M, Rivera J, Rodríguez F, Plana-Durán J, García JA. Protection of rabbits against rabbit hemorrhagic disease virus by immunization with the VP60 protein expressed in plants with a potyvirus-based vector. Virology. 2001;280:283-91.

31. Simon M, Loudet O, Durand S, Bérard A, Brunel D, Sennesal F, et al. Quantitative trait loci mapping in five new large recombinant inbred line populations of Arabidopsis thaliana genotyped with consensus single nucleotide polymorphism markers. Genetics. 2008;178:2253-64.

32. Van Ooijen JW, Voorrips RE. JoinMap ${ }^{\circledR}$ 3.0, Software for the calculation of genetic linkage maps. Wageningen, Netherlands: Plant Research International; 2001

33. Paux E, Faure S, Choulet F, Roger D, Gauthier V, Martinant J, et al. Insertion site-based polymorphism markers open new perspectives for genome saturation and marker- assisted selection in wheat. Plant Biotechnol J. 2010;8:196-210

34. Decroocq V, Favé M, Hagen L, Bordenave L, Decroocq S. Development and transferability of apricot and grape EST microsatellite markers across taxa. Theor Appl Genet. 2003;106:912-22.

35. Atwell S, Huang YS, Vilhjalmsson BJ, Willems G, Horton M, Li Y, et al. Genome-wide association study of 107 phenotypes in Arabidopsis thaliana inbred lines. Nature. 2010;465(7298):627-31.

36. Purcell S, Neale B, Todd-Brown K, Thomas L, Ferreira MAR, Bender D, et al. PLINK: a toolset for whole-genome association and population-based linkage analysis. Am J Hum Genet. 2007;81.

37. Kang HM, Zaitlen NA, Wade CM, Kirby A, Heckerman D, Daly MJ, et al. Efficient control of population structure in model organism association mapping. Genetics. 2008;178:1709-23.

38. Rotenberg D, Thompson T, German T, Willis D. Methods for effective real- time RT-PCR analysis of virus-induced gene silencing. J Virol Methods. 2006;138:49-59.

\section{Submit your next manuscript to BioMed Central and take full advantage of:}

- Convenient online submission

- Thorough peer review

- No space constraints or color figure charges

- Immediate publication on acceptance

- Inclusion in PubMed, CAS, Scopus and Google Scholar

- Research which is freely available for redistribution 